\section{Aligner system with unparalleled results}

orthocaps is an aligner system developed by Dr Wajeeh Khan, an orthodontist practising in Germany.

This German system has been successfully used for over ten years by 2,000 orthodontists and dentist in 35 countries around the world. The system has a unique method of combining two different soft aligners for day and night time use to achieve unparalleled results. These two types differ from one another in both their composition and the amount of pressure they exert. This innovation is called the TwinAligner system.

orthocaps aligners are manufactured with high-performance materials and cutting-edge techniques. The result is an extremely effective method of orthodontic correction. The orthocaps TwinAligner system represents an important step forward - safetywise, aesthetically, and technologically - in the field of orthodontics. It is also able to utilise a hybrid technique of using lingual bracket auxiliaries for complex movements together with aligners to obtain excellent results.

www.orthocaps.com, www.orthoaligners.com

\section{Dental product award winners}

$3 \mathrm{M}$ Oral Care is delighted to have been named a Top Award Winner 2017 by The Dental Advisor Product Awards 2017.

The stand-out products recognised in the Awards were:

- Bonding agent: Universal - Scotchbond universal Adhesive

- Composite: Universal-multilayer - Filtek Supreme XTE universal restorative

- Composite polishers: Posterior - Sof-Lex diamond polishing system

- Impression material: VPS - Imprint 4 VPS impression material

- Post and core kit - RelyX 3D fiber post kit

- Long-term performer: Self-adhesive cement - RelyX Unicem 2 automix self-adhesive resin cement

- Cements: Adhesive resin - RelyX Ultimate adhesive resin cement

- Long-term performer: Provisional - Protemp 4 resin.

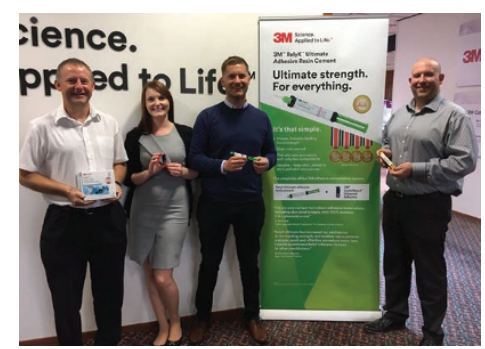

$3 \mathrm{M}$ Oral Care remains dedicated to product research and development. Find out more about the winning solutions and what the judges had to say about them today. For more information, call 08456025094 or visit www.3Mespe.co.uk.

3M, Scotchbond, Filtek, Sof-Lex, Imprint, RelyX and Protemp are trademarks of the $3 \mathrm{M}$ Company.

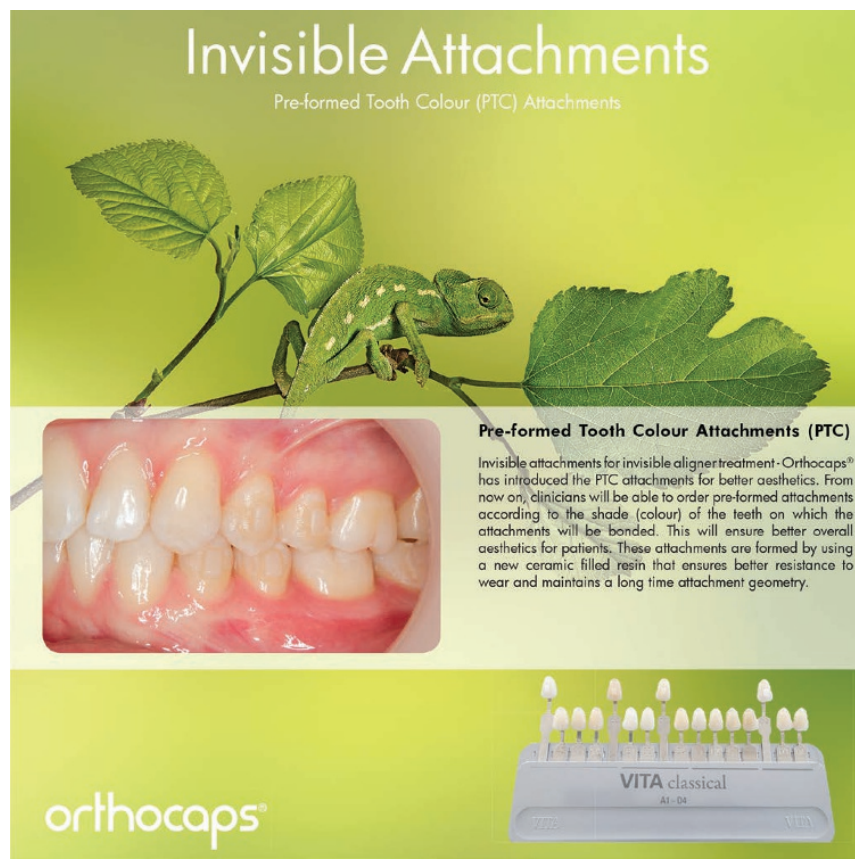

\section{Setting the standard}

A-dec is known as one of the most trusted names in dentistry for good reason. The US-based company produces dental chairs and ensures reliability for dental practice solutions, including upholding the highest standards of service developed from its more than 50-year history.

In its continuing focus on enhancing the skills of its dedicated partners, A-dec conducted its annual A-dec Service Training Course at its UK headquarters in Nuneaton, Warwickshire, over two weeks in July. Under the guidance of a US A-dec trainer, more than 70 engineers earned their stripes as accredited technicians.

The annual training not only contributes to upgrading the standards of the technicians, but also brings peace of mind to clients in the dental industry that products will be long-lasting and easy to maintain. It is all part of the A-dec tradition of providing exemplary after-sales service by a dedicated team.

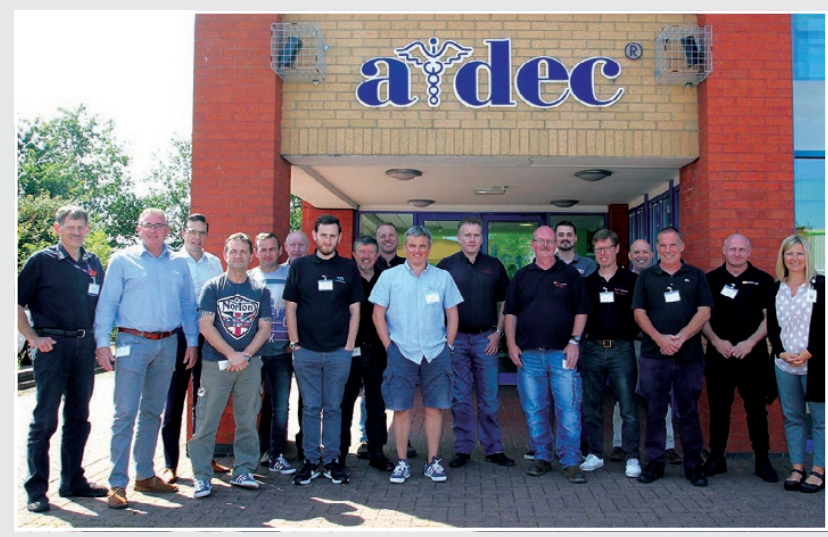

For more information about A-dec Dental UK Ltd, visit www.a-dec.co.uk or call 0800233285. 